\title{
Volatility and Agent Adaptability in a Self-Organizing Market
}

\author{
N.F. Johnson ${ }^{1}$, S. Jarvis ${ }^{1}$, R. Jonson ${ }^{1}$, P. Cheung ${ }^{2}$, Y.R. Kwong ${ }^{2}$, and P.M. Hui ${ }^{2}$ \\ 1 Physics Department, Oxford University, Oxford, OX1 3PU, England \\ 2 Physics Department, The Chinese University of Hong Kong, Shatin, Hong Kong
}

(September 8, 2018)

\begin{abstract}
We present results for the so-called 'bar-attendance' model of market behavior: $p$ adaptive agents, each possessing $n$ prediction rules chosen randomly from a pool, attempt to attend a bar whose cut-off is $s$. The global attendance time-series has a mean near, but not equal to, $s$. The variance, or 'volatility', can show a minimum with increasing adaptability of the individual agents.
\end{abstract}


The dynamical properties of complex adaptive systems are beginning to attract significant attention in many disciplines]. Economics, which historically was based on notions of static equilibria involving rational agents, is now the subject of much of this attention. Of particular interest in the field of Finance are the microscopic factors which can give rise to macroscopic market fluctuations, or 'volatility $\sqrt{3}$ 目.

Arthurl has proposed the so-called 'bar-attendance' model to investigate the global behavior in a market containing heterogeneous agents with bounded rationality acting via inductive reasoning. Specifically $p$ adaptive agents, each possessing $n$ prediction rules or 'predictors' chosen randomly from a pool of $N$, attempt to attend a bar, whose cut-off is $s$, on a particular night each week. Each week the agents update their best rule for predicting a given week's attendance based on the past attendance time-series $x(t)$. This feedback mechanism with its adaptive feature provides an essential ingredient for creating complex dynamics of $x(t)$. A 'mean-field' solution for agent behavior, whereby the majority of agents use a given predictor in a given week, is unstable since it will lead to a large deviation of $x(t)$ from the cut-off $s$ for that week. Other features of interest to a physicist include the fact that the problem lies in the mesoscopic regime in terms of particle (agent) number (i.e. $p \sim 10^{2}$ ), the fact that the interactions between particles (agents) are non-local in time and space, and the fact that the basic bar model's evolution is purely deterministict.

Here we present results of extensive computer simulations for the bar-attendance model. We find that the volatility of the attendance time-series $x(t)$ can show a minimum at small, but finite, $n$. Hence increasing agent adaptability does not generally lead to lower market volatility: in particular it typically increases market volatility, thereby contradicting the idea that well-developed markets with 'expert' traders should be inherently less volatile than emerging markets.

The computer model setup is as follows. The pool of $N$ predictors is chosen to encompass a variety of simple, yet realistic, prediction rules. Unlike the recent works of Challet and Zhang: and Savit et al. 6 , we do not restrict all predictors to depend on the same number of past weeks' data. The predictors in our pool of $N$ are chosen from a variety of 'classes' 
of rule: one class might comprise rules which take an arithmetic (class (i)), geometric (class (ii)), or weighted (class (iii)) average over the past $m$ weeks' attendances; rules from another class (class (iv)) might copy the result from week $\mathrm{m}^{\prime}$; alternatively, the mirror image of $x(t)$ about $s$ might be taken from week $m^{\prime \prime}$ (class (v)). Allowing $m, m^{\prime}$ and $m^{\prime \prime}$ to vary from unity to a few tens, for example, would generate a pool containing $N \approx 400$ rules. We have found that the results of our simulations are quite general provided that a variety of rule-classes are always represented in the pool; this is reasonable since, in real markets, professional analysts will often have quite different recipes for predicting future trends. At the start of a given simulation, each agent chooses $n$ rules from the pool of $N$ (repetitions are allowed) and an arbitrary sequence of attendances is input to simulate earlier weeks' data. We have checked that the long-time behaviour, as indicated by the convergence of the standard deviation of attendance, of the model is generally insensitive to the initial attendance-string. Apart from the predictors, there is an additional difference between the present model and that of Refs 5 and 6: the global parameter representing the weekly outcome in the present model is the actual attendance number, as opposed to a simple binary digit. This allows us to analyse microscopic conditions before an attendance 'crash' or 'bubble'. Such signatures are discussed elsewhere目.

We have studied several weekly update schemes for our basic bar model. One scheme (method I) involves each agent choosing from his $n$ predictors based on the cumulative performance of each of these $n$ predictors: when a given week's attendance $x_{m}$ is known, each agent examines each of his $n$ predictors to see which, in hindsight, would have worked. A cumulative performance can then be assigned to each predictor according either to the decision (i.e. go or stay) implied by the outcome or to the error by which the predictor's outcome differed from $x_{m}$. The predictor with the best cumulative performance is then used to decide the action to be taken in week $m+1$. This scheme introduces a certain 'reluctance to change' - i.e. a predictor with a good track record will not be rejected just because of one bad performance. An alternative scheme (method II) involves the best rule for last week's data being used to predict the outcome of the coming week: when a given week's attendance 
$x_{m}$ is known, each agent examines each of his $n$ predictors to see which, in hindsight, would have worked best. He then uses this model to predict the outcome for week $m+1$. If the predicted outcome is greater than $s$, he stays at home. If it is less than $s$, he attends the bar. If he attends when the actual number attending is less than $s$, he is satisfied. If it is more, he is dissatisfied. We have carried out simulations using both methods and the results are qualitatively similar with some minor differences; in particular, method I gives a distribution of weekly attendance with a sharper peak than method II.

The individual agents' perspective provides a local utility - a given agent is happy with any attendance smaller than $s$ as long as he is one of the attendees. However, large fluctuations represent a wasteage: a negative (positive) fluctuation away from $s$ in any given week implies that he should have attended (stayed away). The individual agents are analogous to traders deciding a day's trading strategy ahead of time, e.g. setting up the computer trades for a given day. For example, imagine each trader (i.e. agent) wishes to buy a given quantity of a particular currency (i.e. he wishes to attend the bar) on a daily basis. Using a simple argument of supply and demand, the price of the currency will rise according to the number of traders (agents) attempting to buy that currency (attend the bar). On a given day, therefore, if too many other traders (agents) try to buy currency (attend the bar) then it will push the price (attendance) above the desirable cutoff value $s$. A large volatility will imply large risk for the trader. The bar-manager has a global utility: for a given mean attendance $\bar{x}$, he will wish to keep the attendance fluctuations (volatility) as small as possible so that he can plan staffing, beer orders etc. He does not, however, care whether an individual agent attends or not. The bar-manager is hence analogous to a government who wishes for a low exchange-rate volatility. A low volatility might therefore be preferable both locally and globally. A detailed analysis of utilities and gains/losses by individual agents are discussed

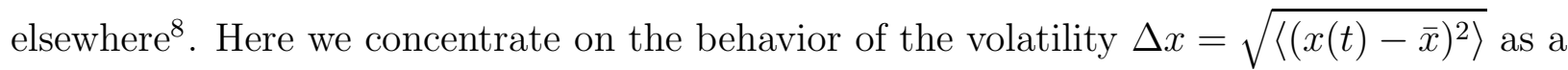
function of $n, p, s$ and $N$.

Figure 1 shows a plot of the volatility $\Delta x$ as a function of the number of predictors $n$ per agent, for the basic bar model. The pool of predictors consists of $N=45$ rules 
from classes (i)-(iv), and method I is used for the weekly updating. There is a minimum in $\Delta x$ for $n \sim 4$. Note that this minimum remains even after 'configurational averaging' (i.e. averaging over an ensemble of simulations with different initial attendance-strings and different distributions of rules among agents). It is also robust against the precise rules contained in the pool $N$, provided that various rule-classes are present as discussed above. The minimum persists when rules of class (v) are also included. The reason for a minimum can be understood as follows. For $n=1$, each agent has no adaptability; even if he is initially dealt a poorly-performing predictor, he has no option but to repeatedly apply it. Increasing $n$ gives him the possibility of replacing this predictor with a better one; $\Delta x$ then decreases with increasing $n$. However now consider the limit $n \rightarrow N$; the agents will hold, on average, increasingly similar toolbags of predictors. In particular, the total attendance will tend to avoid the value $s$, instead forming peaks on either side. The volatility will be large. A minimum in $\Delta x$ at small $n$ is therefore reasonable. The choice of the values of the parameters implies an appreciable overlap of the $n$ rules among the toolbags of the agents: however the overlap is not large enough to cause identical decisions by the agents. It should be noted that for given $n, s$, and $p, \Delta x$ decreases as $N$ increases and eventually saturates to a finite large- $N$ limit.

Figure 2 shows a plot of the volatility $\Delta x$ and mean $\bar{x}$ as a function of the number of agents $p$ for two different values of $N$. For $p<s, \bar{x} \approx p$ and $\Delta x \approx 0$ as expected. For $p>s$, the mean attendance is similar, but not equal, to $s$. On closer inspection we note that for $s<p<2 s, \bar{x}$ is slightly less than $s$ while for $p>2 s, \bar{x}$ becomes greater than $s$. The volatility $\Delta x$ seems to follow a square-root-like dependence on $p$ for $p>s$.

It is tempting to suggest a random walk argument in order to explain the results in Fig. 2. Suppose that the attendence is a stochastic process: specifically imagine it is a first-order Markov process so that the past weeks' data does not contain any extra information for the agent. To compensate for this loss of information, we will supply him with an extra piece of information by giving him the value of $p$ (N.B. In the basic bar model simulation, we did not supply each agent with the value of $p$ ). Given the common knowledge of the cutoff $s$, 
each agent will attend with a probability $\frac{s}{p}$ and stay away with a probability $1-\frac{s}{p}$. Each of the $p$ agents carries out the same calculation, hence the average weekly attendance (i.e. $\bar{x}$ ) should be $p \cdot \frac{s}{p}=s$. Following standard random walk results 9 , the corresponding volatility $\sim \sqrt{4 \cdot p \cdot \frac{s}{p} \cdot\left(1-\frac{s}{p}\right)}$, i.e. $\Delta x \sim \sqrt{4 s\left(1-\frac{s}{p}\right)}$. The solid line on Fig. 2 shows that this is a fairly good approximation. In fact it is better than expected. It is only in the large- $N$ limit that the stochastic, random walk limit should actually be quantitatively reasonable: in the large- $N$ limit the pool is so large that agents are highly unlikely to have any rules in common. The agents therefore are effectively no longer competing against each other in terms of predictors. On closer inspection of this random walk argument, one finds that while the $\sqrt{s\left(1-\frac{s}{p}\right)}$ factor persists, the factor 4 in the expression for $\Delta x$ is not justified given that the walk is confined to the half-space $x \geq 0$; hence the random walk scaling should only be regarded as a qualitative guide. An alternative random walk model can be obtained as follows. Each agent regards the predicted outcome from his set of $n$ predictors as being so complex that he might as well toss a coin when deciding whether to attend in a given week. The probability for attendance is hence 0.5 , leading to $\bar{x} \sim \frac{p}{2}$ and $\Delta x \sim \sqrt{p}$. Although this model captures the steady rise in $\bar{x}$ with $p$ observed in Fig. 2, together with the $\sqrt{p}$-like dependence of $\Delta x$, it is also only useful as a qualitative guide. We have not been able to find a stochastic model which reproduces the quantitative results of the simulations - this is perhaps not surprising given the complexity of the dynamical system being studied.

Figure 3 shows a plot of the volatility $\Delta x$ as a function of cutoff $s$ for $0<s<p$. The qualitative scaling $\sqrt{4 s\left(1-\frac{s}{p}\right)}$ based on random walk arguments is also shown.

Finally, we mention two generalizations of the basic bar model which can be included in an attempt to capture additional features of real agent behavior. A full discussion and results are presented elsewhere 8 .

(i) Evolution and Learning.

Instead of retaining the same $n$ predictors throughout the simulation, each agent will throw back into the pool a predictor which persistently underperforms. He will then randomly 
choose another predictor from the pool. In this way, predictors can become either 'live' or 'dead' during the simulation. Since the pool is of fixed size $N$, the situation can arise where a number of agents begin to use the same successful predictor, hence nullifying its accuracy. This may lead to a 'mass-killing' of this predictor; simultaneously, it allows a predictor that had lain 'dead' in the pool the possibility of being resurrected during the random picking process. Hence we have introduced a degree of evolution and learning into each agent's predictor-set.

(ii) Irrational behaviour in a crowd.

In the basic model, each agent tries to profit from the accuracy of his best model (i.e. the model with minimum error). Here, however, we introduce the feature whereby a small subset of the $N$ predictors are evaluated inaccurately. All agents holding the affected predictors will therefore be misled by the outcomes. This feature is reminiscent of how a rumour spreading through a given subset of traders can affect their decisions and, ultimately, the markets themselves. We find that this feature can give rise to intermittency effects in the volatility $\mathrm{E}$.

In summary we have presented an analysis of the bar-attendance model. This model offers a simple paradigm for a competitive marketplace where agents with bounded rationality act using inductive reasoning. We hope that the present work will stimulate further interest in what is proving to be an exciting field of study for physicists.

One of us (N.F.J.) would like to thank Colin Mayer, David Sherrington, Philippe Binder, Robert Bacon and Jean-Philippe Bouchaud for useful discussions at various stages during this work. This work was supported in part by a grant from the British Council and the Research Grants Council of the Hong Kong SAR Government through the UK-HK Joint Research Scheme 1998. 


\section{REFERENCES}

${ }^{1}$ J. Casti, New Scientist p.36 (13 July, 1996).

2 J.H. Holland, Hidden Order: How Adaptation Builds Complexity, (Addison-Wesley, New York, 1995).

${ }^{3}$ G. Galdarelli, M.Marsili and Y.C. Zhang, Europhys. Lett. 40, 479 (1997).

${ }^{4}$ For two very interesting examples of stochastic models, see R. Cont and J.P. Bouchaud (preprint cond-mat/9712318) and P. Bak, M. Paczuski and M. Shubik Physica A 246, 430 (1997).

${ }^{5}$ D. Challet and Y.C. Zhang, Physica A 246, 407 (1997).

${ }^{6}$ R. Savit, R. Manuca and R. Riolo (preprint adap-org/9712006)

${ }^{7}$ W.B. Arthur, Amer. Econ. Rev. 84, 406 (1994).

${ }^{8}$ P. Cheung, Y.R. Kwong, P.M. Hui, S. Jarvis, R. Jonson and N.F. Johnson (unpublished).

${ }^{9}$ F. Reif, Fundamentals of Statistical and Thermal Physics, p. 22 (McGraw-Hill, New York, 1981). 


\section{Figure Captions}

Figure 1: Volatility $\Delta x$ as a function of the number of predictors $n$ per agent. $N=45$, $p=100$ and $s=60$.

Figure 2: Volatility $\Delta x$ and mean $\bar{x}$ as a function of the number of agents $p . n=5, s=60$ and $N=45$ and 400 . The random walk scaling $\sqrt{4 s\left(1-\frac{s}{p}\right)}$ is also shown.

Figure 3: Volatility $\Delta x$ as a function of the cutoff $s . n=5, s=60$ with $N=45$ and 400. The random walk scaling $\sqrt{4 s\left(1-\frac{s}{p}\right)}$ is also shown (dashed line). 


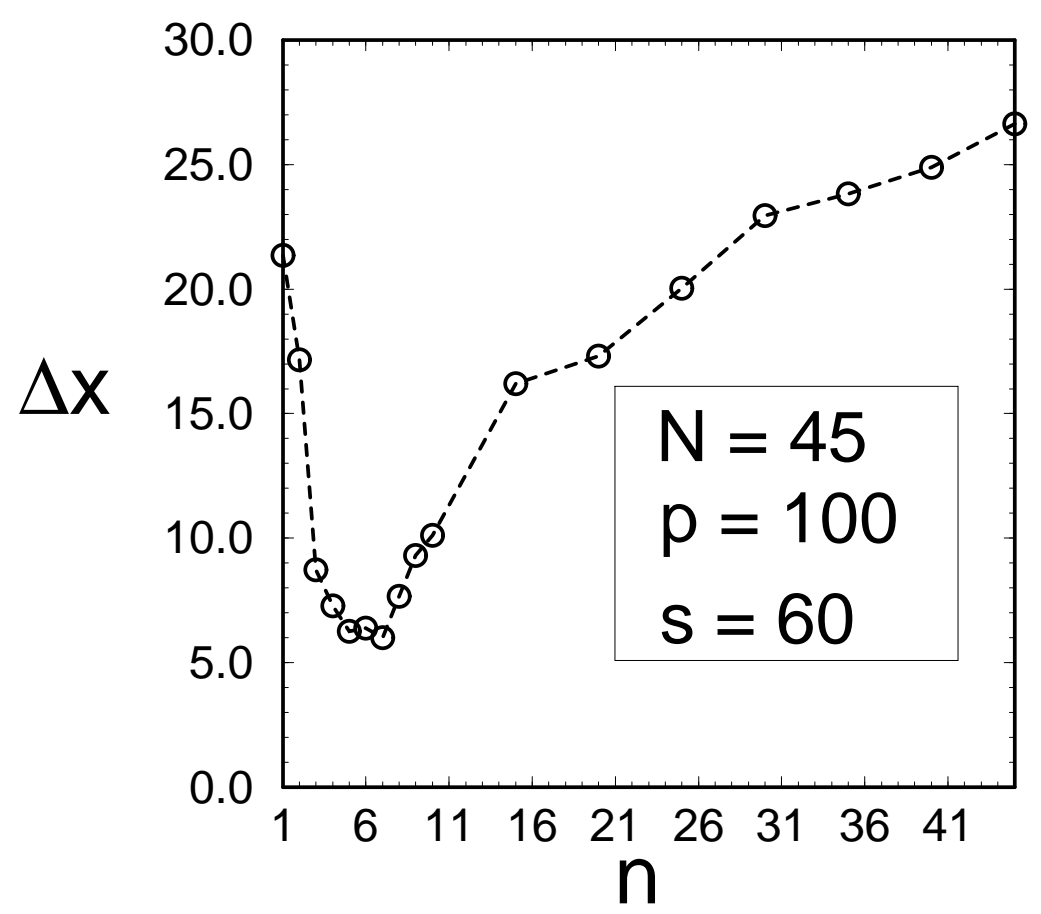




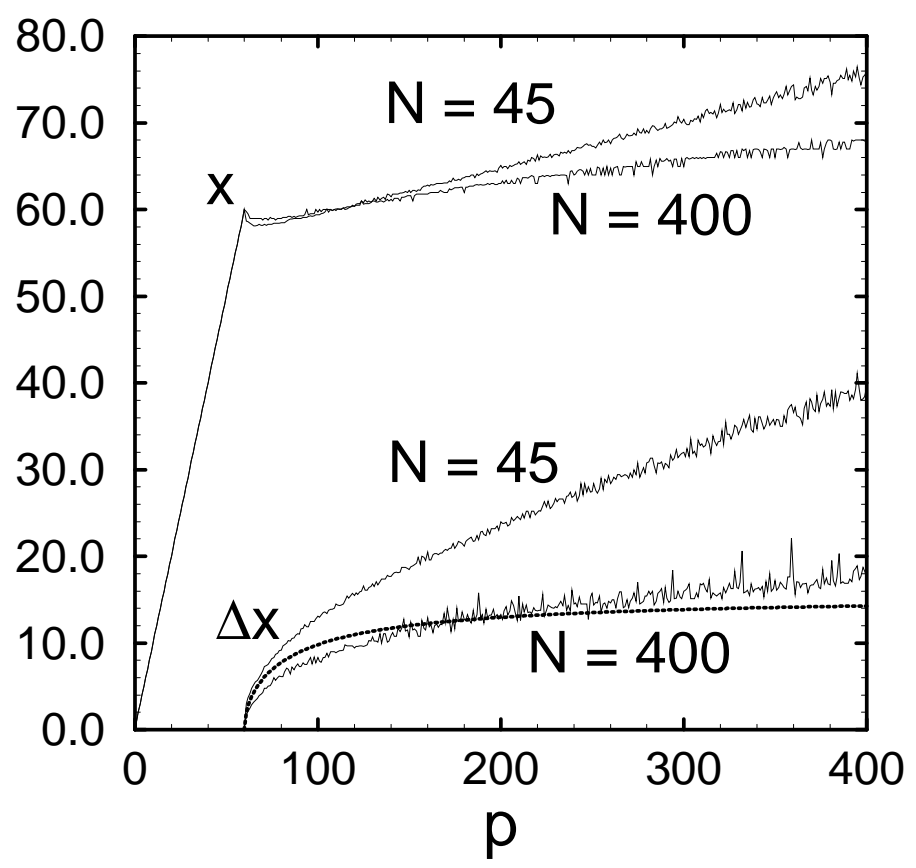




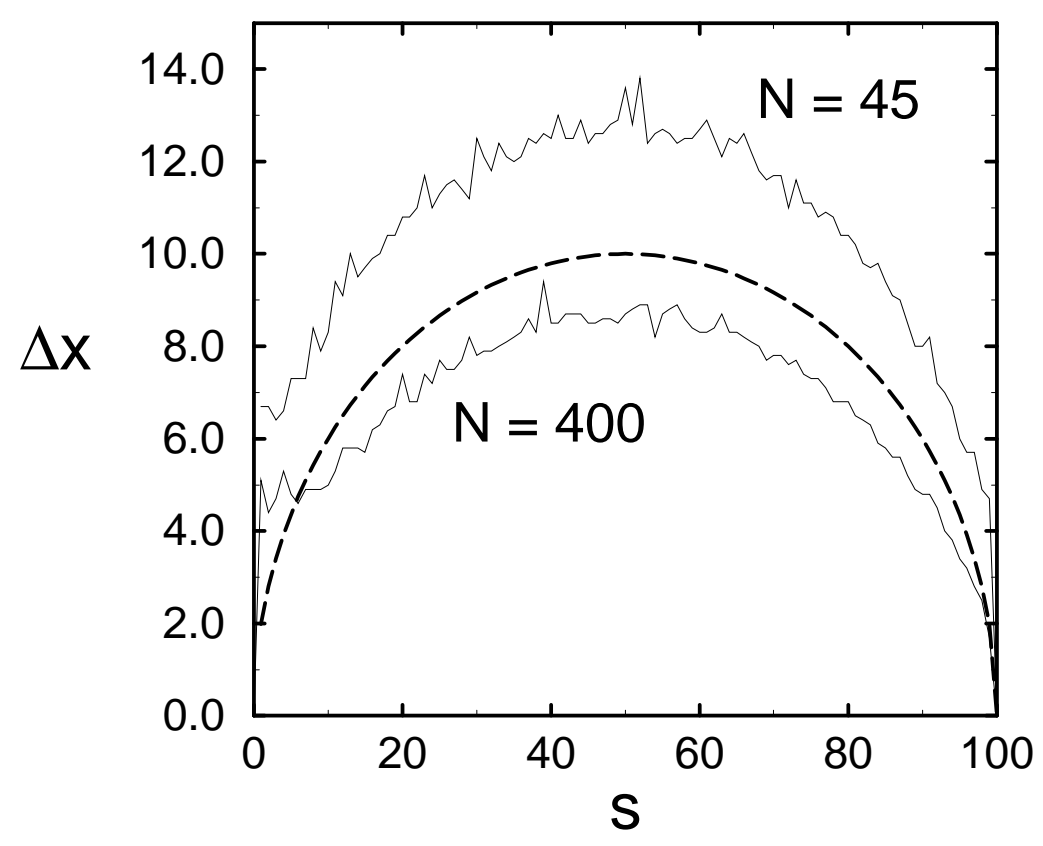

